\title{
Design and implementation of a jellyfish otolith-inspired MEMS vector hydrophone for low-frequency detection
}

\author{
Renxin Wang' ${ }^{1}$ Wei Shen', Wenjun Zhang ${ }^{1}$, Jinlong Song ${ }^{1}$, Nansong Li ${ }^{2}$, Mengran Liư ${ }^{3}$, Guojun Zhang ${ }^{1}$,
} Chenyang Xue ${ }^{1}$ and Wendong Zhang ${ }^{1}$

\begin{abstract}
Detecting low-frequency underwater acoustic signals can be a challenge for marine applications. Inspired by the notably strong response of the auditory organs of pectis jellyfish to ultralow frequencies, a kind of otolith-inspired vector hydrophone $(\mathrm{OVH})$ is developed, enabled by hollow buoyant spheres atop cilia. Full parametric analysis is performed to optimize the cilium structure in order to balance the resonance frequency and sensitivity. After the structural parameters of the $\mathrm{OVH}$ are determined, the stress distributions of various vector hydrophones are simulated and analyzed. The shock resistance of the $\mathrm{OVH}$ is also investigated. Finally, the $\mathrm{OVH}$ is fabricated and calibrated. The receiving sensitivity of the $\mathrm{OVH}$ is measured to be as high as $-202.1 \mathrm{~dB} @ 100 \mathrm{~Hz}(0 \mathrm{~dB} @ 1 \mathrm{~V} / \mu \mathrm{Pa})$, and the average equivalent pressure sensitivity over the frequency range of interest of the $\mathrm{OVH}$ reaches $-173.8 \mathrm{~dB}$ when the frequency ranges from 20 to $200 \mathrm{~Hz}$. The $3 \mathrm{~dB}$ polar width of the directivity pattern for the $\mathrm{OVH}$ is measured as $87^{\circ}$. Moreover, the $\mathrm{OVH}$ is demonstrated to operate under $10 \mathrm{MPa}$ hydrostatic pressure. These results show that the $\mathrm{OVH}$ is promising in low-frequency underwater acoustic detection.
\end{abstract}

\section{Introduction}

Recently, long-distance and weak noise detection for submarines has become a research hot spot, which places high requirements on the low-frequency performance of hydrophones. The submarine acoustic energy of the most powerful discrete components is located in the frequency band of $5-200 \mathrm{~Hz}^{1}$. Vector hydrophones have become a top choice to monitor sound pressure and velocity at low frequency ${ }^{2}$. Many studies have focused on the study of vector hydrophones. Yildiz et al. ${ }^{3}$ developed hydrophone arrays of vector sensors with spacing much less than the wavelength. Ma et al. ${ }^{4}$ reported a two-axis slim fiber laser vector hydrophone with a V-shaped

\footnotetext{
Correspondence: Wendong Zhang (wdzhang@nuc.edu.cn)

'State Key Laboratory of Dynamic Testing Technology, North University of China, Taiyuan, China

${ }^{2}$ College of Underwater Acoustic Engineering, Harbin Engineering University, Harbin, China

Full list of author information is available at the end of the article
}

flexed beam as the mass-spring element. Di Iorio et al. ${ }^{5}$ developed hydrophones to detect cracking sounds through nonintrusive monitoring of bivalve movement. Heerford et al. ${ }^{6}$ introduced a novel fiber hydrophone without any electrical power that can be applied to a hydrophone towed array. The volume of those vector hydrophones is usually large. On the other hand, hydrophones with miniaturized volumes and high sensitivity have become a trend ${ }^{7}$. Lee et al. ${ }^{8}$ presented a kind of MEMS piezoelectric flexural-mode hydrophone with air backing. They also developed a micromachined hydrophone employing a piezoelectric body on the gate of a field-effect transistor ${ }^{9}$. Xu et al. ${ }^{10}$ presented a kind of AlN-on-SOI micromachined hydrophone with high sensitivity. However, these piezoelectric hydrophones were nondirectional and could bear only low hydrostatic pressure due to the sealed membrane structure. This means that the work depth was limited to a low range, which was reported as $100 \mathrm{~m}^{9}$. Ganji et al. ${ }^{11}$ designed a

\section{(c) The Author(s) 2021}

(cc) Open Access This article is licensed under a Creative Commons Attribution 4.0 International License, which permits use, sharing, adaptation, distribution and reproduction cc) in any medium or format, as long as you give appropriate credit to the original author(s) and the source, provide a link to the Creative Commons license, and indicate if changes were made. The images or other third party material in this article are included in the article's Creative Commons license, unless indicated otherwise in a credit line to the material. If material is not included in the article's Creative Commons license and your intended use is not permitted by statutory regulation or exceeds the permitted use, you will need to obtain permission directly from the copyright holder. To view a copy of this license, visit http://creativecommons.org/licenses/by/4.0/. 
MEMS piezoelectric vector hydrophone based on a cilium structure. Amiri et al. ${ }^{12}$ designed a flat cap mushroom-shaped MEMS piezoelectric hydrophone. These kinds of hydrophones were directional and could bear relatively high hydrostatic pressure due to the open structure. However, they have not been fabricated and measured so far, and only the design and simulation have been completed. Generally speaking, MEMS piezoelectric hydrophones are considered more sensitive than other hydrophones, whether nondirectional or directional, measured or in design. The main concern is that the fabricated devices are based on sealed membrane structures, bringing high sensitivity but low work depth. MEMS piezoresistive vector hydrophones have been developed that have the advantages of low-frequency operation and miniaturized detection ${ }^{13}$. They were also proven to be able to resist high hydrophone pressure due to their open structure. Much work has been done in this area, including microstructure parameter optimization, cilium optimization, and package optimization. A whisker-inspired MEMS vector hydrophone (WIVH) was proposed, which was encapsulated with Parylene, in order to improve the sensitivity-frequency response performance $^{14}$. A cup-shaped MEMS vector hydrophone $(\mathrm{CuVH})$ was presented with an improved sensitivity of -209.2 dB@100 Hz (0 dB@1 V/ $\mu \mathrm{Pa})^{15}$. A lollipop-shaped MEMS vector hydrophone ( $\mathrm{LVH})$ was developed, and its sensitivity reached $-205 \mathrm{~dB} @ 100 \mathrm{~Hz}(0 \mathrm{~dB} @ 1 \mathrm{~V} / \mu \mathrm{Pa})^{16}$. How can the performance of vector hydrophones be further improved in the low-frequency range?
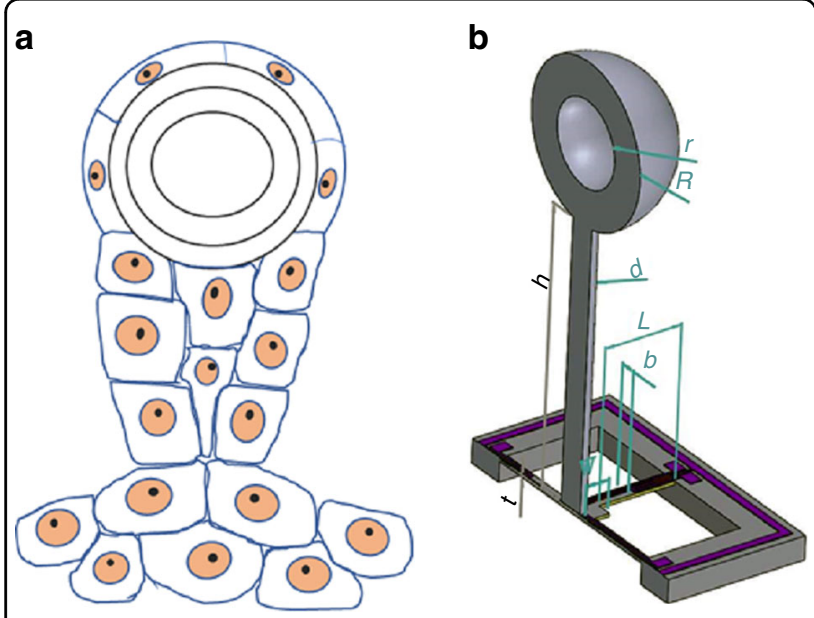

Fig. 1 Microstructure models of auditory organs of pectis jellyfishes and $\mathbf{O V H}$. a Auditory organs of pectis jellyfishes, $\mathbf{b} \mathrm{OVH}$ with an otolith-shaped cilium and cross beam; $b$ is the width of the beam, $t$ is the thickness of the beam, I is the length of the beam, $w$ is the half-width of the mass square, $h$ is the height of the rod, $d$ is the radius of the rod, $R$ is the outer radius of the sphere, and $r$ is the inner radius of the sphere.
Auditory organs of pectis jellyfish consist of otoliths, auditory hairs, and supporting cells, as shown in Fig. 1a. The auditory organ is naturally buoyant and can respond notably strongly to the sound of ultralow frequency. Inspired by the auditory organs of pectis jellyfish, we propose a kind of otolith-inspired MEMS vector hydrophone $(\mathrm{OVH})$ relying on a hollow buoyant sphere on top of the cilium, as shown in Fig. 1b. The bionic microstructure consists of two important parts: a cross-beam structure and a cilium with otolith-shaped microstructure. The cilium is mounted on the center of the cross-beam microstructure, and piezoresistors are located at the root of the beams. When an acoustic wave is applied on the cilium, the cilium vibrates, causing deformation of the cross beam. Finally, the resistances of the piezoresistors change, which is converted to voltage signals via a Wheatstone bridge. Therefore, underwater acoustic signals can be detected.

\section{Materials and methods}

When designing the microstructure, two key problems must be taken into account: increasing the sensitivity and broadening frequency of the band. To address this issue, we must analyze the influence of the microstructure on the sensitivity and frequency bandwidth. In the acoustic-electric transduction process, the cilium plays an important role in the perception of sound waves, which transmit the vibration of medium particles to the microstructure. It is observable that the cilium structure parameters have a great influence on the performance of the hydrophone. A full parametric analysis is performed to optimize the cilium structure. In addition, the hydrophone encounters shock in launching and working procedures. It is also challenging to keep the structure robust to improve the sensitivity. Shock resistance is researched. A sketch of the microstructure dimension is shown in Fig. 1 b.

\section{Influence on the resonance frequency}

The resonance frequency is determined by the energy method. According to the law of conservation of energy, the strain energy $T_{\max }$ and kinetic energy $V_{\max }$ are constant in the case of free vibration without damping:

$$
T_{\max }=V_{\max }
$$

When the cilium is subjected to a horizontal force $F_{x}$, two kinds of moments arise: a flexural moment $M_{x}$ produced by the $X$-axis beam and a torque moment $M_{t}$ produced by the $Y$-axis beam, which remain balanced with an external force $F_{x}$, as shown in Fig. 2a.

It can be assumed that the strain energy of the cilium and kinetic energy of the beam can be neglected. 

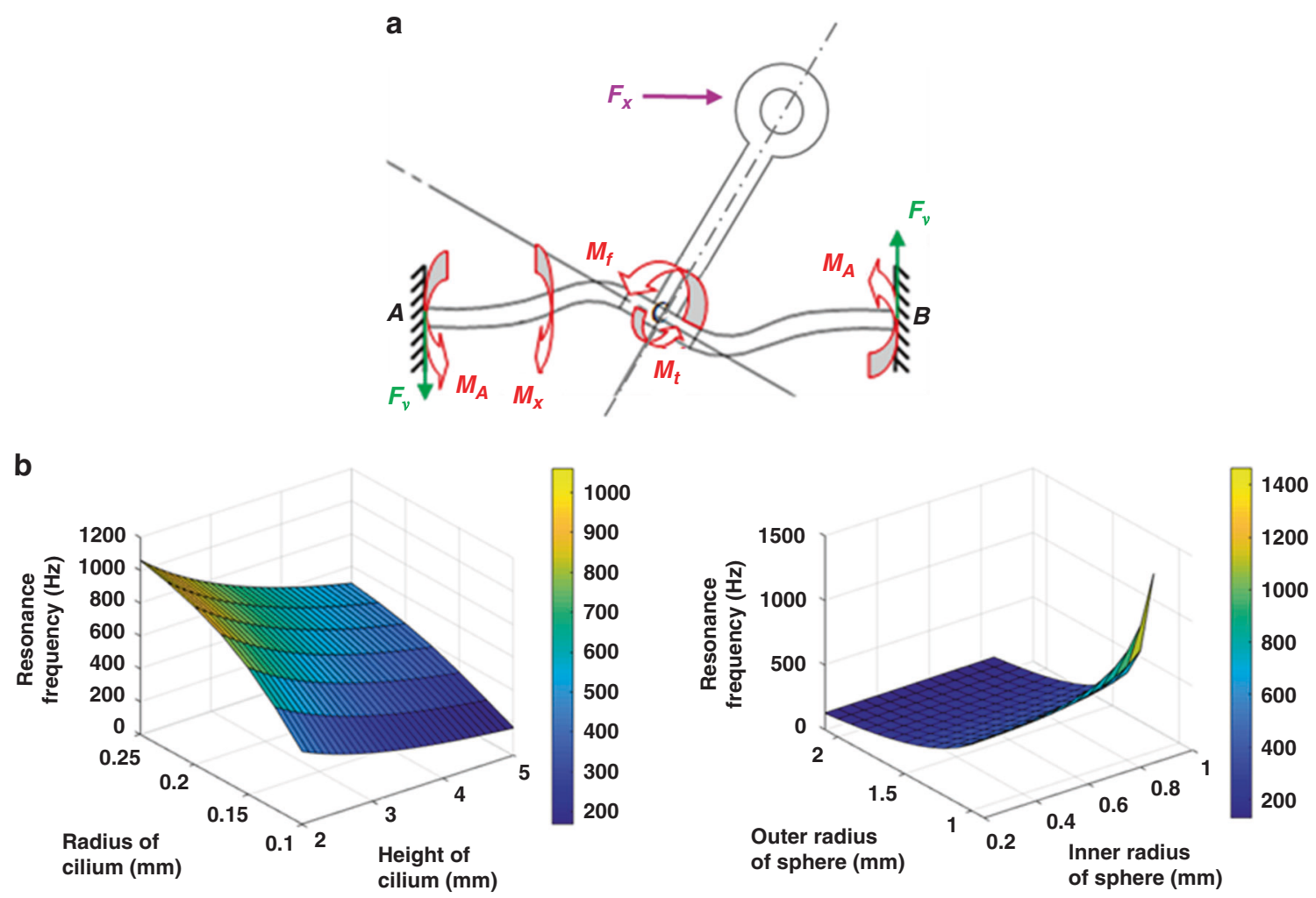

C
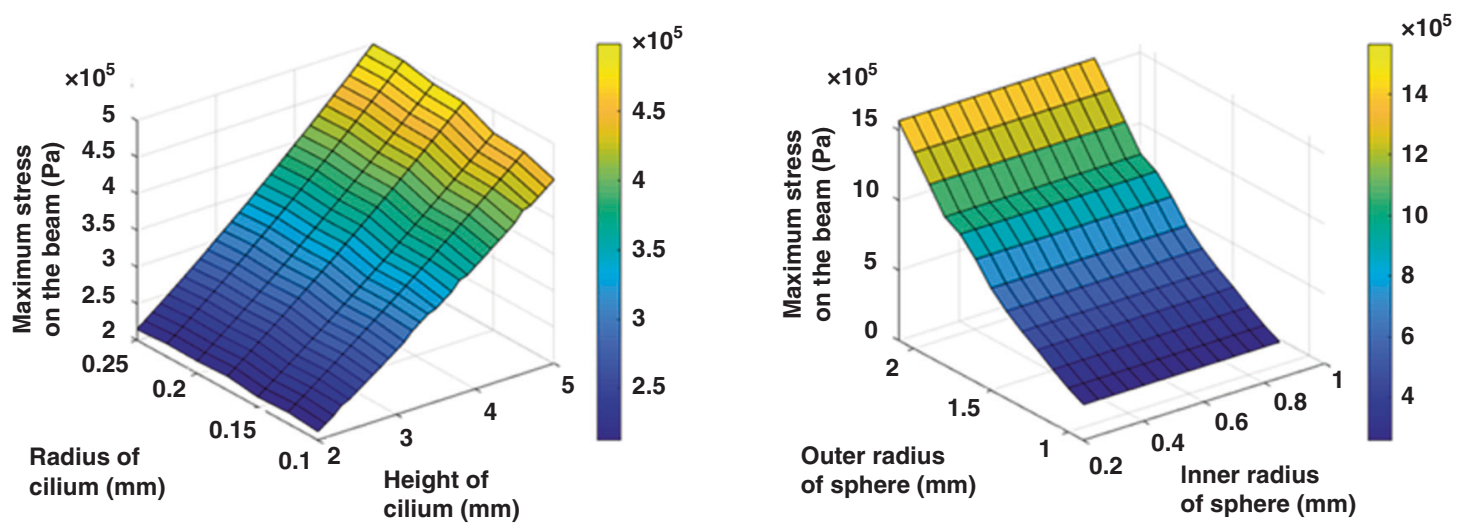

Fig. 2 Analysis of the influence of the structure parameters on the resonance frequency and stress. a Mechanical analysis of the structure; b relationship of the resonance frequency and various structure parameters; c relationship of the maximum stress on the beam and various structure parameters.

The strain energy of the beam consists of the bending energy and torsion energy:

$$
\begin{aligned}
V_{\max } & =2 \times\left(\frac{1}{2} \int_{0}^{l} \frac{M_{x}^{2}}{E I} \mathrm{~d} x+\frac{1}{2} M_{t} \theta_{l}\right) \\
& =\frac{F_{v}^{2} l^{3}+3 F_{v} M_{A} l^{2}+3 M_{A}^{2} l}{3 E I}+\frac{G \beta b t^{3} \theta_{l}^{2}}{l}
\end{aligned}
$$

where $E$ is the elastic modulus of the cantilever beam, $I$ is the moment of inertia, $\theta_{l}$ is the bent angle of the mass square, $G$ is the shear modulus, and $\beta$ is the torsion coefficient.

The kinetic energy of the cilium is as follows:

$$
T_{\max }=\frac{1}{2} \omega^{2}\left[\int_{0}^{h} \rho \pi d^{2}\left(y \theta_{l}\right)^{2} \mathrm{~d} y+\rho \times \frac{4}{3} \pi\left(R^{3}-r^{3}\right)(h+R)^{2} \theta_{l}^{2}\right]
$$

The relationship of the resonance frequency and various structure parameters is demonstrated in Fig. 2b, which is obtained by modal analysis. 


\section{Influence on the sensitivity}

Assuming that an external pressure $P_{x}$ is applied on the cilium along the $X$-direction, the beam is bent and compressed. The stress distribution on the cantilever beam is:

$$
\begin{aligned}
\sigma_{x}(x)= & \frac{3(1+v)\left[-(3 l+6 w) x+\left(l^{2}+3 w l\right)\right]}{2 b t^{2}\left[(1+v)\left(l^{2}+3 w l+3 w^{2}\right)+6 \beta\left(5 l^{2}+12 w l\right)\right]} \\
& {\left[d h^{2}+\pi R^{2}(h+R)\right] P_{x}+\frac{\left(2 d h+\pi R^{2}\right) P_{x}}{b t} }
\end{aligned}
$$

where $v$ is the Poisson ratio of the beam and $x$ is the distance to the beam root.

The piezoresistors are distributed on the root of the beams, which are nearly the location of maximum stress. The output voltage of the Wheatstone bridge is approximately proportional to the maximum stress on the beam:

$$
\begin{aligned}
V_{o \_}= & \left\{\frac{3(1+\nu)\left(l^{2}+3 w l\right)}{2 b t^{2}\left[(1+\nu)\left(l^{2}+3 w l+3 w^{2}\right)+6 \beta\left(5 l^{2}+12 w l\right)\right]}\right. \\
& {\left.\left[d h^{2}+\pi R^{2}(h+R)\right]+\frac{\left(2 d h+\pi R^{2}\right)}{b t}\right\} P_{x} \pi_{l} V_{\mathrm{in}} }
\end{aligned}
$$

where $\pi_{l}$ is the piezoresistance coefficient and $V_{\text {in }}$ is the input voltage. It can be concluded that the stress distribution is related to the size parameters of the microstructure.

Pressure loads are applied along the $Y$-direction, and the stress distribution curves with different parameters can be obtained by static analysis. The relationship of the maximum stress on the beam and various structure parameters is shown in Fig. 2c.

Taking the resonance frequency and maximum stress into consideration, the dimensions of the microstructure are illustrated in Table 1. The resonance frequency of the microstructure is $527 \mathrm{~Hz}$ in air and $314 \mathrm{~Hz}$ in water, resulting in a working bandwidth as high as $200 \mathrm{~Hz}$ (an analysis is shown in the Supplementary File). Moreover, the stress is optimized to realize high sensitivity. The stress nephogram on the microstructure is illustrated in Fig. 3a. The stress distributions can be obtained from the nephogram. Compared to the case of the previously presented $\mathrm{LVH}, \mathrm{CuVH}$, and $\mathrm{WIVH}$, the maximum stress of the $\mathrm{OVH}$ is obviously higher, as shown in Fig. 3b.

\section{Influence on the shock resistance}

When the hydrophone encounters shock with acceleration $a$, the beam is bent and compressed due to the inertia force of the cilium $f_{c}$ (the inertia force of the beam can be neglected). The maximum stress of the structure appears at the root of the silicon beam and does not exceed the fracture strength of $\mathrm{Si}(175 \mathrm{MPa})$.

When OVH encounters $X$-direction shock with acceleration $a_{x}$, the stress analysis is similar to that of the sensitivity. In view of the shape of the cilium, the moment of inertia force is divided into two parts: the sphere and the rod, due to the different lengths of the force arm:

$$
\begin{aligned}
\sigma_{\mathrm{x} \_ \text {max }}= & \frac{3(1+\nu)\left(l^{2}+3 w l\right)}{2 b t^{2}\left[(1+\nu)\left(l^{2}+3 w l+3 w^{2}\right)+6 \beta\left(5 l^{2}+12 w l\right)\right]} \\
& {\left[\frac{1}{2} d^{2} h^{2}+\frac{4}{3}\left(R^{3}-r^{3}\right)(h+R)\right] \pi \rho a_{x} } \\
& +\frac{\left[d^{2} h+\frac{4}{3}\left(R^{3}-r^{3}\right)\right] \pi \rho a_{x}}{b t}
\end{aligned}
$$

When the OVH encounters a $Z$-direction shock with acceleration $a_{z}$, the inertia force of cilium is vertically applied on the central square, resulting in beam bending:

$$
\sigma_{z_{-} \max }=\frac{3\left(2 l^{2}+2 b l+b w\right)}{4 b t^{2}(2 l+b)}\left[d^{2} h+\frac{4}{3}\left(R^{3}-r^{3}\right)\right] \pi \rho a_{z}
$$

Stress nephograms were simulated via Comsol Multiphysics (Fig. 4), in order to find the maximum stresses of the $\mathrm{OVH}$ structures at an acceleration of $60 \mathrm{~g}$ along the $X$ - and $Z$-directions, which were 115.8 and $12.5 \mathrm{MPa}$, respectively. In contrast, the corresponding maximum stresses of the LVH were 181.4 and $34.7 \mathrm{MPa}$. The corresponding maximum stresses of the $\mathrm{CuVH}$ were 151.7 and $18.6 \mathrm{MPa}$. The maximum stresses of the WIVH along the $X^{-}$and $Z$-directions were 38.7 and $4.2 \mathrm{MPa}$,

Table 1 Dimensions of the microstructure.

\begin{tabular}{llll}
\hline Dimension & Value & Dimension & Value \\
\hline Outer radius of the sphere $(\mu \mathrm{m})$ & 1000 & Inner radius of the sphere $(\mu \mathrm{m})$ & Height of the rod $(\mu \mathrm{m})$ \\
Radius of the rod $(\mu \mathrm{m})$ & 175 & Length of the beam $(\mu \mathrm{m})$ & 330 \\
Thickness of the beam $(\mu \mathrm{m})$ & 40 & Width of the mass square $(\mu \mathrm{m})$ & 1000 \\
Width of the beam $(\mu \mathrm{m})$ & 120 & 600 \\
\hline
\end{tabular}



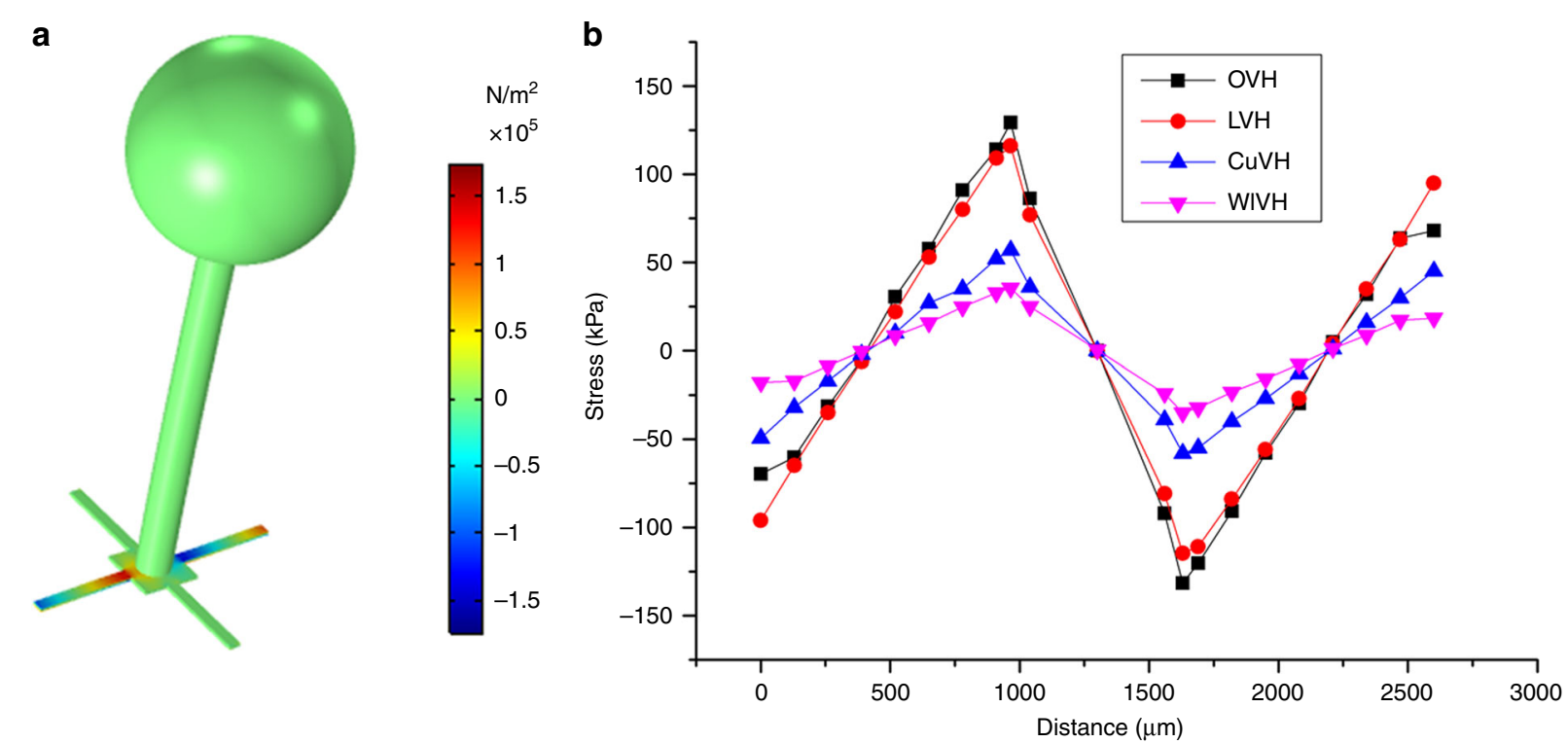

Fig. 3 Simulation and comparison of the stress on the microstructures. a Stress nephogram on the cross beam of the OVH when external pressure is applied on the cilium along the $X$-direction. $\mathbf{b}$ Stress distributions on the beams of various structures. Values of the $X$-axis indicate the distance of the site on the beam from the starting point.

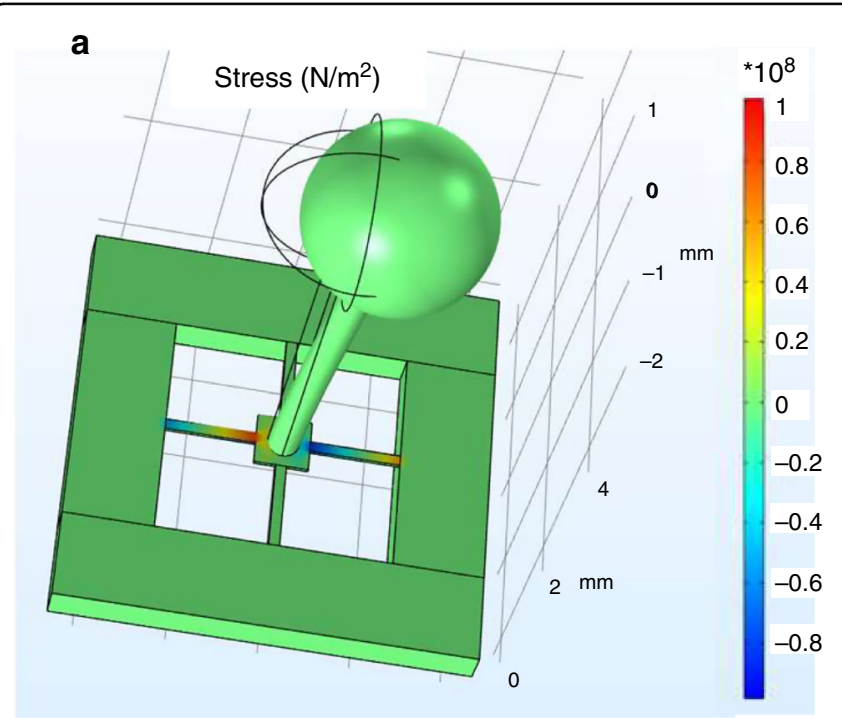

b

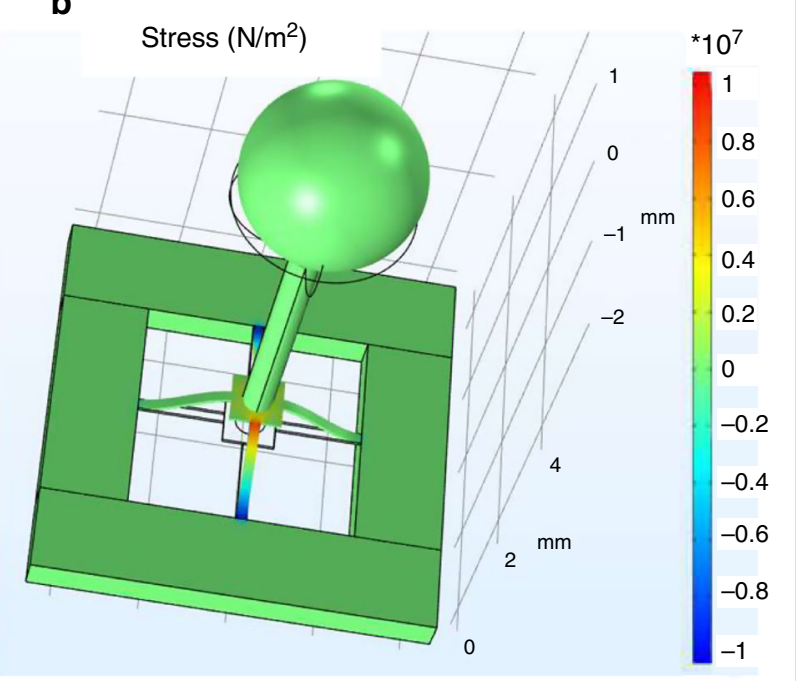

Fig. 4 Stress nephogram under $60 \mathrm{~g}$ shock along diverse directions. a Along the $X$-direction; $\mathbf{b}$ along the $Z$-direction. The maximum stress on the beam could be extracted as $115.8 \mathrm{MPa}$ along the $X$-direction and 12.5 .

respectively. In contrast with the structure with the stereo cilium such as the $\mathrm{OVH}, \mathrm{LVH}$, and $\mathrm{CuVH}$, the maximum stress of the WIVH was lower. It should be noted that the maximum stress of the WIVH was lower than that of the $\mathrm{OVH}$ by $9.5 \mathrm{~dB}$, but the average equivalent pressure sensitivity of the WIVH was lower by $13.6 \mathrm{~dB}$, as shown in Table 2. In addition, the OVH could resist a higher shock than the $\mathrm{LVH}$ and $\mathrm{CuVH}$. It should be noted that the sensitivity of the $\mathrm{OVH}$ was higher than that of the LVH and $\mathrm{CuVH}$ as a result of the otolith-shaped microstructure. On the one hand, this design provides a high receiving area and moment of force when the sound wave is intercepted. On the other hand, hollow spheres can reduce the influence of shock. 


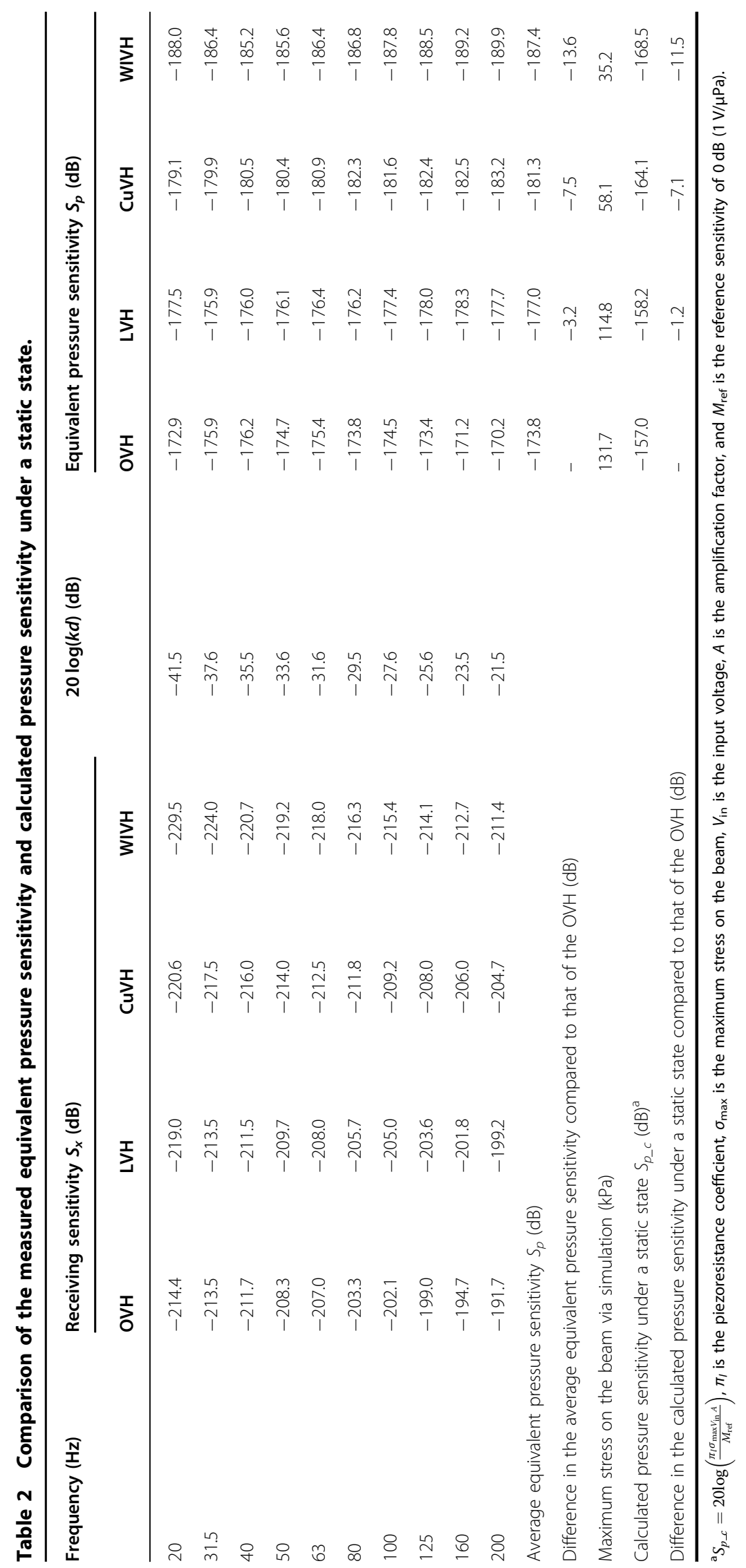




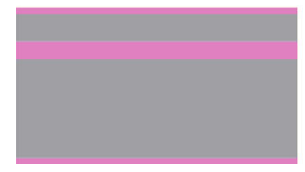

(1) Thermal oxidation on SOI

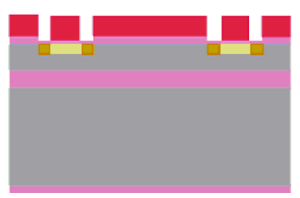

(4) 2nd lithography, boron heavy implantation,

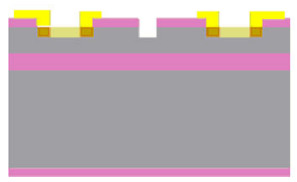

(7) 4th lithography, forward shallow etch

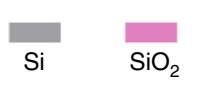

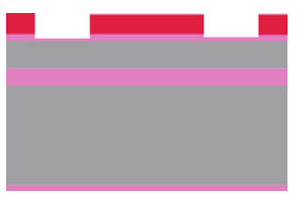

(2) 1st lithography, etch $\mathrm{SiO}_{2}$ with $40 \mathrm{~nm}$ residue

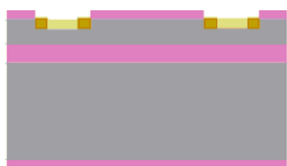

(5) Remove surface $\mathrm{SiO}_{2}$ anneal, remove PR

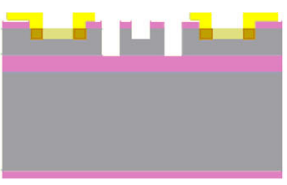

(8) 5th lithography, forward through etch

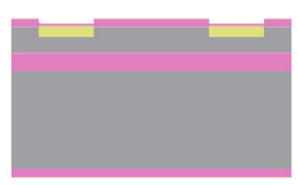

(3) Boron light implantation, remove $\mathrm{PR}$

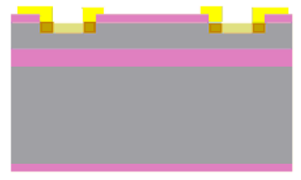

(6) Sputter, 3rd lithography, etch, anneal to form ohm contact

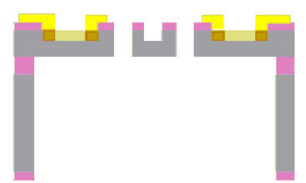

(9) 6th lithography, backside through etch, release

Fig. 5 Sketch of the microfabrication process. (1) Thermal oxidation; (2) 1st lithography, Etch $\mathrm{SiO}_{2}$ with 40 nm residue; (3) Boron light implantation, remove photoresist; (4) 2nd lithography, Boron heavy implantation; (5) Remove surface $\mathrm{SiO}_{2}$, anneal, remove photoresist; (6) Sputter, 3rd lithography, etch the metal, anneal to form Ohm contact; (7) 4th lithography, forward shallow etch; (8) 5th lithography, forward through etch; (9) 6th lithography, backside through etch, release the structure.

\section{Microfabrication process of the $\mathrm{OVH}$}

The critical component of the MEMS hydrophone is the cross beam, where the piezoresistors are distributed. The dimensional parameters of the cross beam have a direct influence on the performance of the hydrophone. Hence, the cross beam is fabricated via the MEMS manufacturing process. The specific process is illustrated in Fig. 5.

\section{Calibration method}

To verify the feasibility of the OVH, a sensitivity/ directivity test is accomplished in a standing wave calibration system. The output voltage of the OVH is compared with that of a reference hydrophone to obtain the sensitivity of the $\mathrm{OVH}$, which is calculated by:

$$
M_{x}=\frac{e_{x}}{P} \frac{\sin k d_{r}}{\cos k d_{M}}
$$

where $M_{x}$ is the sensitivity, $e_{x}$ is the output voltage of the hydrophone, $k$ is the wavenumber $(k=\omega / c), \omega$ is the circular frequency, $c$ is the sound wave velocity in water, and $d_{M}$ and $d_{r}$ are the distances from the water surface to the $\mathrm{OVH}$ and the reference hydrophone, respectively. $P$ can be obtained by measuring the output voltage of the reference hydrophone ${ }^{17,18}$.
Furthermore, the receiving sensitivity of MEMS hydrophone $S_{x}$ is given by:

$$
S_{x}=20 \log \left(\frac{e_{x} / p}{M_{\text {ref }}} \tan k d\right)
$$

Here, $M_{\text {ref }}$ is the reference sensitivity as $0 \mathrm{~dB}(1 \mathrm{~V} / \mu \mathrm{Pa})$, and $d$ is the distance from the water surface to the vector hydrophone and reference hydrophone, both of which are set at the same distance.

In this experiment, $d$ is set as $0.1 \mathrm{~m}$. The frequency ranges from 20 to $200 \mathrm{~Hz}$, corresponding to $k d \in[0.009,0.088]$, where $\tan (k d) \approx k d$ according to Taylor's formula:

$$
\begin{aligned}
S_{x}=20 \log \left(\frac{e_{x} / p}{M_{\mathrm{ref}}} k d\right) & =20 \log \left(\frac{e_{x} / p}{M_{\mathrm{ref}}}\right)+20 \log (k d) \\
& =S_{p}+20 \log (k d)
\end{aligned}
$$

$S_{p}$ can be considered the equivalent pressure sensitivity of the MEMS hydrophone. 
a

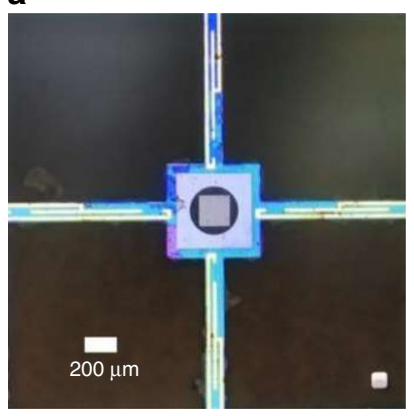

C

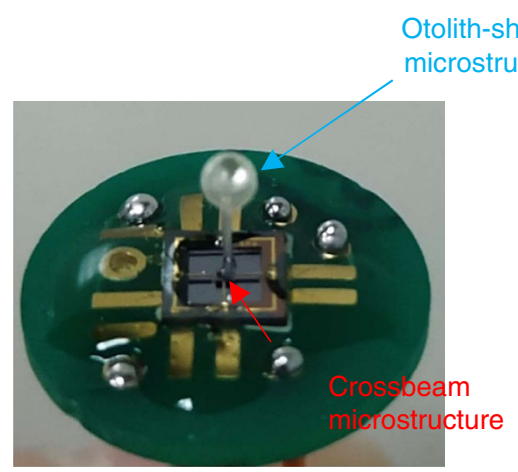

d

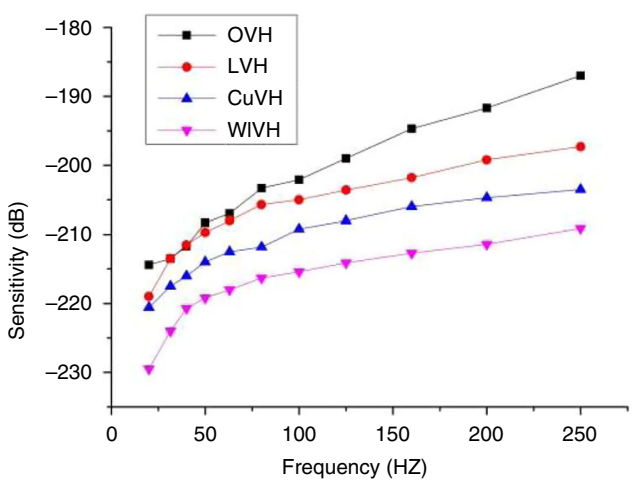

f

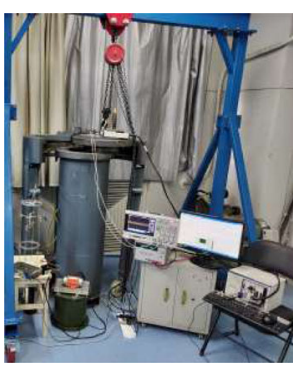

b

Otolith-shaped
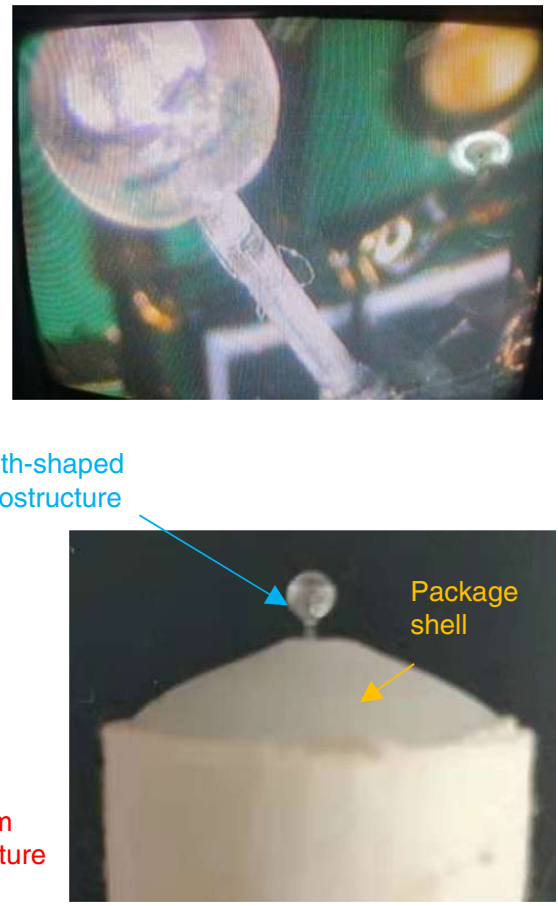

e

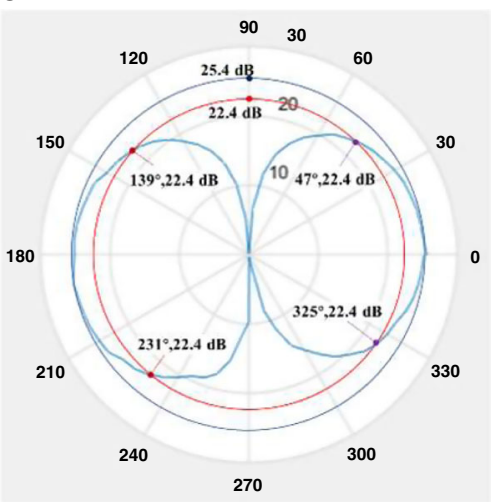

h
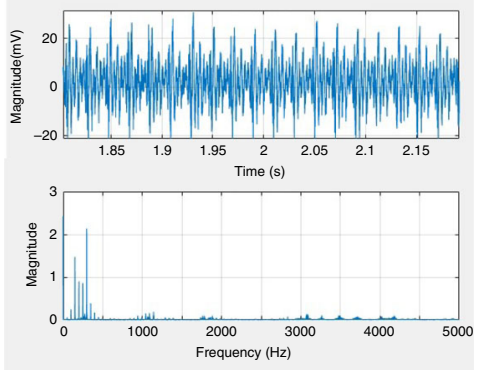

Fig. 6 Measurement results of the OVH. a Microscopy photograph of the cross-beam microstructure. Cross beam, piezoresistors, metal lines, and shallow groove can be seen; $\mathbf{b}$ picture of an otolith-shaped cilium mounted on a beam; $\mathbf{c}$ picture of the chip on the PCB and in the shell; the otolithshaped cilium has been mounted vertically on the center of the cross beam; $\mathbf{d}$ receiving sensitivity-frequency response curve; $\mathbf{e}$ directivity pattern at $100 \mathrm{~Hz}$ with $3 \mathrm{~dB}$ polar width of the $\mathrm{OVH}$ at $87^{\circ}$; $\mathbf{f}$ setup of the $10 \mathrm{MPa}$ hydrostatic pressure measurement; $\mathbf{g}$ otolith-shaped microstructure after the $10 \mathrm{MPa}$ test; $\mathbf{h}$ data acquisition under $10 \mathrm{MPa}$ 


\section{Results}

A microscopy photograph of the microstructure is shown in Fig. 6a. The cross beam is formed in the suspending state. The piezoresistors are distributed on the beam. The metal lines are intact. The shallow groove at the center can be observed, which is favorable in cilia alignment and integration.

The otolith-shaped microstructure, with a hollow buoyant sphere on the top of the rod, was manufactured by microprecision $3 \mathrm{D}$ printing equipment based on projection microstereolithography technology (BMF microArch P130, up to $2 \mu \mathrm{m}$ resolution). Otolith-shaped cilia with UV-curable glue were mounted on the central hole of the cross beam by a customized alignment setup, as shown in Fig. 6b.

A physical photograph of the OVH is shown in Fig. 6c. Three-micrometer-thick Parylene was deposited conformally on the cilium and beam by SCS PDS 2010 to ensure electrical insulation and structural protection. The otolith-shaped cilium was mounted vertically on the center of the cross beam. Finally, the chip with the processing circuit was packaged in a shell.

The receiving sensitivity-frequency response curves of various MEMS hydrophones are shown in Fig. 6d. The equivalent pressure sensitivity $S_{p}$ over the frequency range of interest can be calculated thorough Formula (8), which is illustrated in Table 2. It can be seen that the average equivalent pressure sensitivity $S_{p}$ over the frequency range of interest of the $\mathrm{OVH}$ reaches $-173.8 \mathrm{~dB}(0 \mathrm{~dB} @ 1 \mathrm{~V} / \mu \mathrm{Pa})$, an increase of $3.2 \mathrm{~dB}$ compared with that of the $\mathrm{LVH}, 7.5 \mathrm{~dB}$ compared with that of the $\mathrm{CuVH}$, and $13.6 \mathrm{~dB}$ compared with that of the WIVH. The measurement results are in accordance with the simulation results extracted from Fig. $3 \mathrm{~b}$, as shown in Table 2 . The absolute values of the calculated pressure sensitivity in the static state deviate from those of the measured average equivalent pressure sensitivity. This may be because calculation at the static state ignores the influence of dynamic damping. This deviation may also be attributed to the effect of package structure, noise of the reference hydrophone, and misestimation of the piezoresistance coefficient. Further investigation on the differences between the theoretical and experimental results should be performed. It should be noted that the differences in the calculated pressure sensitivity at the static state compared to the case of the $\mathrm{OVH}$ are consistent with those of the measured average equivalent pressure sensitivity.

The directivity pattern of the $\mathrm{OVH}$ at $100 \mathrm{~Hz}$ is shown in Fig. 6e, exhibiting typical cosine directivity. The $3 \mathrm{~dB}$ polar width of the $\mathrm{OVH}$ is measured as $87^{\circ}$, which shows superiority compared with $96^{\circ}$ for the $\mathrm{LVH}, 89^{\circ}$ for the $\mathrm{CuVH}$, and $91^{\circ}$ for the WIVH. This means that the OVH would perform better in distinguishing the sound along the sensitive axis than the LVH and slightly better than the $\mathrm{CuVH}$ and the WIVH.
Measurement under hydrostatic pressure was performed, as shown in Fig. 6f, including the equipment to implement $10 \mathrm{MPa}$ hydrostatic pressure and data acquisition. The vibration motor was mounted to the hydrostatic pressure tube and utilized as a stimulating source. With $10 \mathrm{MPa}$ hydrostatic pressure applied on the $\mathrm{OVH}$, the otolith-shaped microstructure maintained its original shape without transformation, as shown in Fig. 6g. Data were acquired when the $\mathrm{OVH}$ was under the environment of $10 \mathrm{MPa}$ hydrostatic pressure and vibration motor operation. As shown in Fig. 6h, the vibration signal could be distinguished in the time zone, and the peak appeared at $297.3 \mathrm{~Hz}$ in the frequency zone, which was in accord with the resonance frequency of the $\mathrm{OVH}$. These results indicate that the $\mathrm{OVH}$ could work well under $10 \mathrm{MPa}$, owing to the open structure and Parylene encapsulation.

\section{Discussion and conclusions}

In this paper, optimizations of cilium structure are made in order to realize high-sensitivity and low-frequency underwater acoustic detection, resulting in an otolith-shaped microstructure. Different parameters of the cilium structure influence the stress distribution and resonance frequency, which are analyzed via a theoretical model and simulation. Stress distributions of the different hydrophones are contrasted through simulations. Shock-resistance analysis shows that the OVH can resist higher shock than the $\mathrm{LVH}$ owing to otolith-shaped microstructure. Then, the fabrication process of the $\mathrm{OVH}$ is demonstrated. Finally, the $\mathrm{OVH}$ is tested in a standing wave field. The results show that the average equivalent pressure sensitivity $S_{p}$ over the frequency range of interest of the $\mathrm{OVH}$ reaches $-173.8 \mathrm{~dB}(0 \mathrm{~dB} @ 1 \mathrm{~V} / \mu \mathrm{Pa})$, an increase of $3.2 \mathrm{~dB}$ compared with that of the $\mathrm{LVH}, 7.5 \mathrm{~dB}$ compared with that of the $\mathrm{CuVH}$, and $13.6 \mathrm{~dB}$ compared with that of the WIVH. Additionally, the OVH has a cosine directional pattern with a $3 \mathrm{~dB}$ polar width of $87^{\circ}$.

Measurement under hydrostatic pressure indicates that $\mathrm{OVH}$ could be feasible under $10 \mathrm{MPa}$, owing to the open structure and Parylene encapsulation. The test results agree with the theoretical and simulation analysis, which verifies the feasibility and advancements of the $\mathrm{OVH}$ in detecting weak low-frequency underwater acoustic signals.

\section{Acknowledgements}

This work was supported by the National Natural Science Foundation of China (Grant 51875535 and 61727806) and by 1331KSC, State Key Laboratory of Precision Measuring Technology and Instruments (pilab1805). The authors are grateful to BMF Material Technology Inc. for their cooperation on microprecision 3D printing and thank Mr. Songxiang Ji and Mrs. Shasha Yang for their help in hydrostatic pressure measurement.

\footnotetext{
Author details

${ }^{1}$ State Key Laboratory of Dynamic Testing Technology, North University of China, Taiyuan, China. ${ }^{2}$ College of Underwater Acoustic Engineering, Harbin Engineering University, Harbin, China. ${ }^{3}$ Hubei Key Laboratory of Modern Manufacturing Quantity Engineering, School of Mechanical Engineering, Hubei University of Technology, Wuhan, Hubei, China
} 


\section{Author contributions}

R.W. and W.D.Z. proposed the idea for the study. W.S. and W.J.Z. fabricated the sensors and performed the testing. J.S. carried out the resonance frequency and stress simulations. R.W. and G.Z analyzed the data. R.W. and N.L. established the theoretical models. R.W. and W.S. wrote the manuscript. M.L. and C.X. provided advice in fabrication and measurement. All the authors discussed the results and the manuscript.

\section{Conflict of interest}

The authors declare that they have no conflict of interest.

Supplementary information accompanies this paper at https://doi.org/ 10.1038/s41378-020-00227-w.

Received: 24 September 2019 Revised: 22 September 2020 Accepted: 19 October 2020

Published online: 01 January 2021

\section{References}

1. Testa, C. \& Greco, L. Prediction of submarine scattered noise by the acoustic analogy. J. Sound Vib. 426, 186-218 (2018).

2. McConnel, J. A. Analysis of a compliantly suspended acoustic velocity sensor. J. Acoust. Soc. Am. 113, 1395-1405 (2003).

3. Yildiz, S., Dorman, L. M. \& Kuperman, W. A. Using hydrophones as vector sensors. J. Acoust. Soc. Am. 135, 2361-2364 (2014).

4. Ma, R., Zhang, W. T. \& Li, F. Two-axis slim fiber laser vector hydrophone. IEEE Photon. Techol. Lett. 23, 335-337 (2011).

5. Di lorio, L., Gervaise, C. \& Jaud, V. Hydrophone detects cracking sounds: nonintrusive monitoring of bivalve movement. J. Exp. Mar. Biol. Ecol. 432, 9-16 (2014).

6. Heerfordt, A., Mohl, B. \& Wahlberg, M. A wideband connection to sperm whales: a fiber-optic, deep-sea hydrophone array. Deep Sea Res. / 54, 428-436 (2007).
7. Palanisamy, P., Kalyanasundaram, N. \& Swetha, P. M. Two-dimensional DOA estimation of coherent signals using acoustic vector sensor array. Signal Process. 92, 19-28 (2012).

8. Lee, H., Choi, S. \& Moon, W. A micro-machined piezoelectric flexuralmode hydrophone with air backing: benefit of air backing for enhancing sensitivity. J. Acoust. Soc. Am. 128, 1033 (2010).

9. Sung, M., Shin, K. \& Moon, W. A micro-machined hydrophone employing a piezoelectric body combined on the gate of a field-effect transistor. Sens. Actuator. A Phys. 237, 155-166 (2016).

10. Xu, J., Zhang, X., Fernando, S. N., Chai, K. T. \& Gu, Y. AlN-on-SOI platform-based micro-machined hydrophone. Appl. Phys. Lett. 109 3-8 (2016).

11. Ganji, B. A., Nateri, M. S. \& Dardel, M. Design and modeling of a novel high sensitive MEMS piezoelectric vector hydrophone. Microsyst. Technol. 24 2085-2095 (2018)

12. Amiri, P., Kordrostami, Z. \& Ghoddus, H. Design and simulation of a flat cap mushroom shape microelectromechanical systems piezoelectric transducer with the application as hydrophone. IET Sci. Meas. Technol. 14, 157-164 (2020).

13. Xue, C. Y., Chen, S. \& Zhang, W. D. Design, fabrication, and preliminary characterization of a novel MEMS bionic vector hydrophone. Microelectron 38 1021-1026 (2007)

14. Wang, R. et al. Wide-frequency-bandwidth whisker-inspired MEMS vector hydrophone encapsulated with parylene. J. Phys. D. Appl. Phys. 49, 07LT02 (2016).

15. Xu, W. et al. Development of cup-shaped micro-electromechanical systemsbased vector hydrophone. J. Appl. Phys. 120, 124502 (2016).

16. Liu, Y. et al. 'Lollipop-shaped' high-sensitivity microelectromechanical systems vector hydrophone based on Parylene encapsulation. J. Appl. Phys. 118 044501 (2015).

17. Gordienko, V. A., Gordienko, E. L. \& Dryndin, A. V. Absolute pressure calibration of acoustic receivers in a vibrating column of liquid. Acoust. Phys. 40, 219-222 (1994).

18. Hong, L. J., Yang, D. S. \& Shi, S. G. Study on a medium three dimensional cooscillating vector hydrophone. J. Vib. Shock 30, 79-84 (2011). 